\title{
The Pressure Enhances the Extraction Yield of Total Triterpenoids from Ganoderma Lucidum
}

\author{
Peilong SUN ${ }^{1, a}$,Yuqiang WANG ${ }^{1, \mathrm{~b}}$ and Wen $\mathrm{TANG}^{1, \mathrm{c}^{*}}$ \\ ${ }^{1}$ School of Perfume and Aroma Technology, Shanghai Institute of Technology,100 Haiquan Road, \\ Fengxian District, Shanghai 201418,P. R. China \\ apeilong.sun@yahoo.com, ${ }^{b} 1002250493 @ q q . c o m,{ }^{* c}$ tangw@sit.edu.cn
}

\begin{abstract}
The high pressure used as a single factor was applied to extract triterpenoids from ganoderma lucidum. In this paper, the conditions of HPCL-Recator extraction were optimized, the optimum extraction parameters were respectively as following: $90^{\circ} \mathrm{C}, 1 \mathrm{Mpa}, 30 \mathrm{~min}, 50 \mathrm{ml} / \mathrm{g}, 70 \%$ $(\mathrm{v} / \mathrm{v})$ ethanol, and the highest extraction yield of triterpenoids reached $1.41 \%$ $(\mathrm{mg} / \mathrm{g})$. Ultrasonic-assited extraction (UAE), microwave-assisted extraction (MAE) were also optimized, and the the highest extraction yield were $1.08 \%$ and $1.21 \%$. The results showed that the high pressure extraction is more efficient than the conventional UAE and MAE.
\end{abstract}

Keywords: high pressure; triterpenoids; extraction; ganoderma lucidum

\section{Introduction}

Ganoderma Lucidum is an important and ancient medical fungus in traditional China, it has been used to strengthen healthy and treat disease[1], Ganoderma Lucidum has multiple bioactive and medicinal activities. Many bioactive components have been identified, triterpenoids are the mainly bioactive compounds, more than 100 triterpenoids have been isolated and they showed antioxidant, immunomodulatory[2]. However, the content of triterpenoid saponins in ganoderma lucidum is limited, thus, a efficient and convenient extraction method is necessary and valuable.

The common extraction methods for ganoderma lucidum are UAE, MAE. UAE has been widely used for extraction from plant material[3], UAE improves extraction yield and reduces extraction time. MAE is seen as an new method and used widely [4], however, MAE can not attain plenty of triterpenoid saponins one time. Compared with UAE and MAE, the high pressure compact laboratory reactors(HPCL-Reactor) is convenient and more effective, because of its large volume, it can acquire a large amount of triterpeniod saponins . High pressure has been used to extract many natural compounds, including flavoniods[5], polyphenols[6], these studies indicated that the pressure was effective for extraction. In this study, by pumping the argon into HPCL-Reactor to increase the inner pressure, which is an inactive gas. The main factors of HPCL-reactor extraction are temperature, pressure, ethanol concentration, extraction time and ratio of solvent to material,

\footnotetext{
* Corresponding author: tangw@sit.edu.cn
} 
we optimized the relevant extraction conditions and compared the extraction yield for the aim of the higher extraction yield of triterpenoids.

\section{Materials and Methods}

\subsection{Materials and reagents}

Dried Ganoderma Lucidum was purchased from Changbai Mountain Protection and Development Zone. Ganoderma Lucidum was sliced and ground into fine powder before extraction.

Ethanol, ethyl acetate, acetic acid, perchloric acid were purchased from Shanghai Chemical Reagent Co. Oleanolic acid and vanillin were purchased from Shanghai Baoman Biological Technology Co.All the reagents were analytical grade.

\subsection{Apparatus}

Ultrasonic bath (Crest Ultrasonics Shanghai Ltd.CP1200D). Microwave extractor (Shanghai PreeKem Scientific Instruments Ltd. WX-6000). Water bath (Shanghai Boxun Industry \& Commerce Co). UV/Vis spectrophotometer (Shanghai Precision \& Scientific Instrument Co. UV759CRT). HPCL-Reactor (Series 5500 HP Compact Reactors) was manufactured by Parr Instrument Company, it includes a pressure and temperature display.

\subsection{Ultrasonic extraction}

UAE was carried out in an ultrasonic bath that allows variations of irradiation power, the extraction factors include extraction time $(15-120 \mathrm{~min})$, rate of solvent to material $(10-50 \mathrm{ml} / \mathrm{g})$, ethanol concentration $(50-90 \%)$, irradiation power $(100-1000 \mathrm{w})$ were optimized in a closed conical flask at room temperature, the extraction solutions were vacuum filtered and stored at $4^{\circ} \mathrm{C}$.

\subsection{Microwave-assisted extraction}

MAE was performed in a closed vessel unit, it was equipped with temperature and pressure sensor, which helps to control temperature accurately. The extraction factors include rate of solvent to material $(10-50 \mathrm{ml} / \mathrm{g})$, ethanol concentration $(50-90 \%)$, duration (15-120min), temperature $\left(50-100{ }^{\circ} \mathrm{C}\right)$, oven power $(200-1000 \mathrm{w})$ were optimized, the extraction solutions were vacuum filtered and stored at $4^{\circ} \mathrm{C}$.

\subsection{HPCL-Reactor extraction}

The extraction of HPCL-Reactor equipped a temperature and pressure sensor was carried out in the thermostatic water bath, the temperature was contained $\pm 2^{\circ} \mathrm{C}$, the pressure was contained $\pm 0.1 \mathrm{Mpa}$. The extraction conditions include rate of solvent to material $(10-100 \mathrm{ml} / \mathrm{g})$, ethanol concentration $(50-90 \%)$, extraction time $(15-120 \mathrm{~min})$, temperature $\left(50-100^{\circ} \mathrm{C}\right)$, the pressure $(0-8 \mathrm{Mpa})$ were optimized, after extraction, the HPCL-Reactor was cooled to room temperature and released the argon, the solutions were vacuum filtered and stored at $4^{\circ} \mathrm{C}$.

\subsection{Analytical methods}

The determination of the total content of triterpenoids was conducted as described by [7] with slight modifications. In briefly, the principle of the method is the reaction of oxidized triterpenoid saponins with vanillin, perchloric acid is the strong oxidant and the distinctive 
color of this reaction is purple and the maximum ultraviolet absorption is near $550 \mathrm{~nm}$. The standard curve was obtained as follows: a stock solution of oleanolic acid $(1 \mathrm{mg} / \mathrm{ml})$ was prepared, than, the different volumes of $12.5,25,50,100,200 \mathrm{ul}$ of the stock solution were transferred into $10 \mathrm{ml}$ test tubes. After the solvent was evaporated out in water-bath, $0.2 \mathrm{ml}$ fresh mixed $5 \%(\mathrm{w} / \mathrm{v})$ vanillin-acetic acid solution and $0.8 \mathrm{ml}$ perchloric acid were added, mixed and incubated at $70^{\circ} \mathrm{C}$ for $20 \mathrm{~min}$. Then, the tubes were taken out and cooled to room temperature, $5 \mathrm{ml}$ of ethyl acetate was added, the absorbance was scanned with a blank solution as reference and the maximum absorption was at $548 \mathrm{~nm}$. The standard line was $C=0.0185 A(\mathrm{R} 2=0.9997)$, where $\mathrm{C}$ is the concentration of triterpenoid saponins of solution $(\mathrm{mg} / \mathrm{ml}), A$ is the absorbance. $0.2 \mathrm{ml}$ filtered extraction solution was addded into a tube and was determined described above. The extraction yield of triterpene saponins $(\%)$ =weight of triterpenoids/weight of simple $\times 100 \%$.

\section{Result and Discussion}

\subsection{Effect of extraction temperature}

Fig. 1 shows the effect of temperature on extraction of triterpenoids, the temperature was from $50^{\circ} \mathrm{C}$ to $100^{\circ} \mathrm{C}$. The extraction yield increased with the increase of temperaure until $90^{\circ} \mathrm{C}$, the highest yield was obtained about $1.11 \%$ at $90^{\circ} \mathrm{C}$, once the temperature was higher than $90^{\circ} \mathrm{C}$, the extraction yield slowly decreased, but the yield was still higher at $100^{\circ} \mathrm{C}$ than that at low temperature, such as $50,60^{\circ} \mathrm{C}$. High temperature is favorable for desorption of triterpenoids from matrix, and enhances penetration of particles [8]. Solvent have higher capacity to solubilize analytes at high temperature, but too high temperature lead to degradation of triterpenoid [9] therefore, $90^{\circ} \mathrm{C}$ was chosen as the optimal temperature for the fllowing experiment.

\subsection{Effect of ethanol concentration}

Ethanol was widedly used for extractions, because of less toxic and easy acquisition. Fig. 2 shows the effect of ethanol concentration, ethanol concentration greatly influenced the extraction yield, when the concentration increased from $50 \%$ to $70 \%$, the extraction yield increased from $0.71 \%$ to $1.28 \%$, this may be due to that triterpenoid was ethanol-soluble. When the ethanol concentration was higher than $70 \%$, the extraction yield was about $1.2 \%$ and decreased slowly. Thus, $70 \%$ was used for subsequent study.

\subsection{Effect of rate of solvent to material}

In this study, volume of solvent must be enough to ensure the samples were entirely immersed. Fig. 3 shows the effect of rate of solvent to material. When the rate of solvent to material was less than $50 \mathrm{ml} / \mathrm{g}$, the extraction yield of triterpenoids increased with the increase rate of solvent/material, the highest extraction yield was obtained with $1.31 \%$, the reason may be that more solvent can enter cells and more analytes can permeate into solvent at larger rate of solvent to material [10]. When the rate of solvent to material was higher than $50 \mathrm{ml} / \mathrm{g}$, the yield of triterpenoid was about $1.34 \%$ and kept steady, while a large volume of solvent increases the yield of triterpenoids, but the yield didn't increase significantly $(\mathrm{P}>0.05)$, so we selected $50 \mathrm{ml} / \mathrm{g}$ as the suitable rate of solvent to material for the further study. 


\subsection{Effect of extraction time}

Fig. 4 shows the effect of time. Extraction yield reached its maximum at $30 \mathrm{~min}$, which was about $1.35 \%$, with the increase of time, the yield slightly decreased, it may be due to the fact that triterpenoid easily decomposed when they were kept at high temperature for a long time [11], for the cost and shorten time, 30min was seen as the best time for extraction.

\subsection{Effect of the pressure}

Fig. 5 shows the effect of the pressure. When the pressure increased from 0Mpa to $1 \mathrm{Ma}(0 \mathrm{Mpa}$ means a normal atmosphere without addition of argon), the extraction yield increased from $0.91 \%$ to $1.38 \%$, it increased significantly. When the pressure was higher than $1 \mathrm{Mpa}$, the extraction yield kept steady, which was about $1.37 \%$. The pressure enhanced the extraction yield, the reason may be that high pressure increased the solubility, according to mass transfer theory [12], the rate of mass transfer= pressure/resistance of mass transfer, the more solvent can fast enter the cell and more compounds can permeate the cell membrane under high pressure. High pressure can greately increase the eatraction yield of triterpenoid, but too high pressure was not safety and expensive, so $1 \mathrm{Mpa}$ was chosed for extraction.

\subsection{Effect of different temperature and pressure}

Fig. 6 shows the extraction yield at different temperature and pressure, the extraction yield was not significantly changed at low temperature with pressure or without pressure, but the yield increased significantly when the pressure increased from $0 \mathrm{Mpa}$ to $1 \mathrm{Mpa}$ at high temperature $(\mathrm{p}<0.05)$, it means the pressure can significantly improve the yield at high temperature.
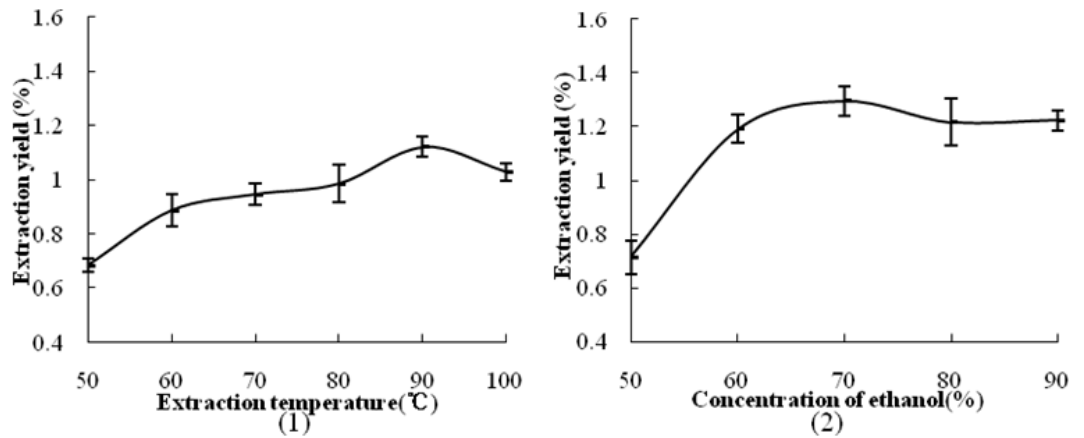

(2)
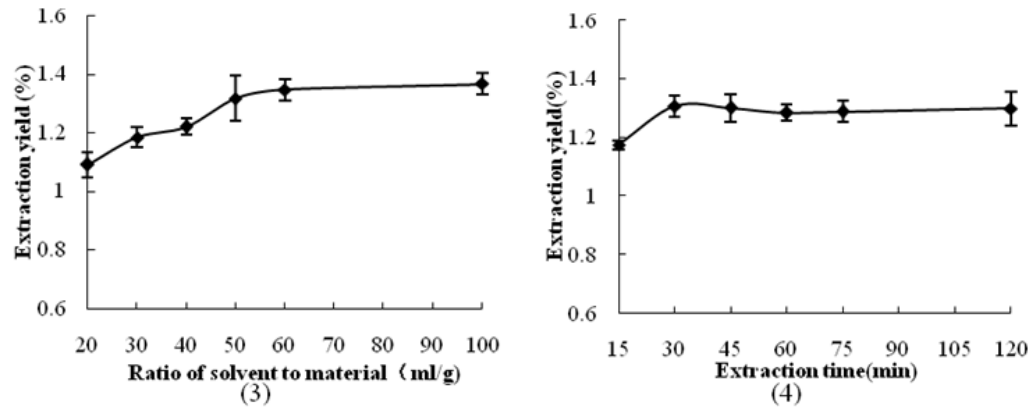

(4) 

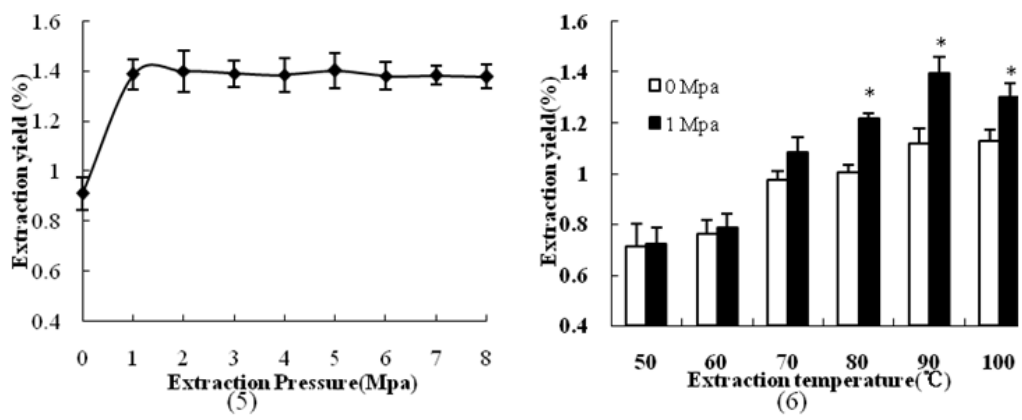

Fig. 1 Effect of temperature on the extraction yield of triterpenoids in ganoderma lucidum (the conditions: $50 \%$ solvent, $2 \mathrm{~g}$ powder, $1 \mathrm{Mpa}$ and $30 \mathrm{~min}$ ). Fig. 2 Effect of ethanol concentrations on the extraction yield of triterpenoids in ganoderma lucidum (the conditions: $100 \mathrm{ml}$ solvent, $2 \mathrm{~g}$ powder, $1 \mathrm{Mpa}, 90^{\circ} \mathrm{C}$ and $30 \mathrm{~min}$ ). Fig. 3 Effect of ratio of solvent to material(the conditions: $70 \%$ solvent, $1 \mathrm{Mpa}, 90^{\circ} \mathrm{C}$, and $30 \mathrm{~min}$ ). Fig. 4 Effect of extraction time(the conditions: $70 \%$ solvent, $2 \mathrm{~g}$ powder, $1 \mathrm{Mpa}$ and $90^{\circ} \mathrm{C}$ ). Fig. 5 Effect of extraction pressure(the conditions: $70 \%$ solvent, $2 \mathrm{~g}$ powder, $90^{\circ} \mathrm{C}$ and $30 \mathrm{~min}$ ). Fig. 6 Extraction yield at different temperatures and pressures ( the condition: $70 \%$ solvent, $2 \mathrm{~g}$ powder, $30 \mathrm{~min}$. ${ }^{*} 0 \mathrm{Mpa}$ compared with $\left.1 \mathrm{Mpa}, \mathrm{p}<0.05\right)$. Datas are the mean $\pm \mathrm{SEM}$.

\section{Comparison of HPCL-Reactor Extraction, UAE and MAE}

Extraction conditions of UAE and MAE were optimized, the largest extraction yield of UAE and MAE were $1.08 \%$ and $1.21 \%$, the extraction yield was lower than the extraction yield of HPCL-Reactor, of which the yield was $1.41 \%$. UAE mainly depends on the ultrasonic energy, the solid and liquid molecules are vibrated quickly, then, the triterpenoid saponins can rapidly come out. MAE mainly depends the direct effect of microwaves on molecules, the solvent and Ganoderma Lucidum particle are heated rapidly when absorb the microwaves, so MAE can obtain high extraction yield in short time, and in this study, we found that ganoderma lucidum could be carbonized, which leads to a lot of impurity, the quality and quantity of triterpenoids would be damaged. Compared with UAE and MAE, HPCL-Reactor extraction was more efficient, and the vessel was much bigger, so HPCL-Reactor extraction can obtain plenty of triterpenoids one time. Besides, HPCL-Reactor is equipped with a stirring equipment, which can also enhance the extraction yield, so the efficiency of HPCL-Reactor can be larger.

\section{Conclusions}

HPCL-Reactor was used to extract triterpenoids, the highest yield was about $1.41 \%$. High pressure extraction was the most efficient extraction methods among UAE and MAE. Most of high pressure extractions were conducted at room temperature, and the pressure ranged from $100 \mathrm{Mpa}$ to $800 \mathrm{Mpa}[13]$. In this study, the temperature was $90^{\circ} \mathrm{C}$, the pressure ranged from $1 \mathrm{Mpa}$ to $8 \mathrm{Mpa}$, the operation was more convenient and safe. Extraction is the first essential step for the isolation and purification of many bioactive compounds from natural products, HPCL-Reactor extraction is suitable for rapid and large-scale extraction, so HPCL-Reactor extraction for triterpenoids is very valuable, and the biological activity experiments of triterpenoids should be conducted in the further study. 


\section{References}

1. T. Yun, Update from Asia: Asian studies on cancer chemoprevention. Ann N Y Acad Sci. 889(1999)157-192.

2. Z. Xu, X. Chen, F. Zhang, Ganoderma Lucidum Polysaccharides: Immunomodulation and Potential Anti-Tumor Activities.AJM. 39 (2011)15-27.

3. C. Leonellia, T. Masonb, Microwave and ultrasonic processing: now a realistic option for industry. Chem Eng Process. 49(2010)885-900.

4. Y. Youn, Y. Ming, S. Yuan, Microwave-assisted extraction of ginsenosides from ginseng root. Microchem J. 71(2003)131-139.

5. S. Zhang, J. Xi, C. Wan, Effect of high hydrostatic pressure on extraction of flavoniods in propolis. IJFST. 11(2005)213-216.

6. J. Xi, d. Shen, S. Zhao, Characterization of polyphenols from green tea leaves using a high hydrostatic pressure extraction. Int J Pharm. 382(2009)139-143.

7. R. Chen, F. Meng, S. Zhang, Effects of ultrahigh pressure extraction conditions on yields and antioxidant activity of ginsenoside from ginseng. Sep Purif Technol. 66(2009)340-346

8. J. Li, Y. Zu, Y. Fu, Optimization of microwave-assisted extraction of triterpene saponins fromdefatted residue of yellow horm kernel and evaluation of its antioxidant activity. Innov Food Sci Emerg Technol. 11(2010)637-643

9. K. Nagendra, B. Prasad, M. Zhao, Effects of high pressure treatment on the extraction yield, phenolic content and antioxidant activity of litchi (Litchi chinensis Sonn.) fruit pericarp. Int J Food Sci Technol. 44(2009)960-966.

10. Q. Zhang, R. Chen, C. Wang, Experiment study of on ultrahigh pressure extraction of Ginsenosides. J Food Eng. 11(2007)79:1-5.

11. R. Richard, High Pressure Phase Behaviour of Multicomponent Fluid Mixtures. Elsevier(2012)

12. H. Yan, Separation Engineering. China Petrochemical Press, Beijing (2002).

13. S. Zhang, J. Zhu, C. Wang, Novel high pressure extraction technology. Int J Pharm. 278(2004) 471-474. 